\title{
A call to arms against ultra-rare diseases
}

\author{
The not-for profit foundation $n$-Lorem seeks to provide experimental antisense oligonucleotide treatments to \\ patients with ultra-rare disease free for their entire lifetime.
}

\author{
Stanley T. Crooke
}

established the n-Lorem charitable foundation in January 2020 with the mission of bringing experimental antisense oligonucleotide (ASO) therapies to patients with extremely rare diseases. It is committed to providing these treatments to patients free of charge, for life. The charity defines patients with ultra-rare diseases as having a mutation unique to them (an $N$ of 1 ) or having a mutation that has been identified as causing disease in fewer than 30 patients worldwide and is therefore out of the reach of traditional commercial drug programs. Though each patient may be unique, the total number of patients afflicted by such rare mutations is thought to be in the millions, and this number grows daily as the genomes of more patients are characterized ${ }^{1}$. The extreme rarity of such patients presents unique challenges to the diagnosis, determination of the genetic cause of disease, and traditional treatment paradigms. Furthermore, the diseases that these patients experience are typically severe, often rapidly fatal and frequently cause developmental delays or arrests. In short, $N$-of- 1 patients are desperate and underserved.

Now that $\mathrm{n}$-Lorem has completed its first year of operation, in this Comment, I provide the rationale for the establishment of the foundation and summarize progress.

I also discuss the scope of ultra-rare disease, the unique needs of $N$-of- 1 patients, the attributes required of a technology that can meet the needs of some of these patients, and the necessity for a new therapeutic model. Although n-Lorem has already made substantial progress in its first year, many challenges remain to reduce the burden and suffering of ultra-rare diseases. Here I discuss the progress made by the foundation and its plans to meet these ongoing challenges.

\section{Building a bridge}

The simplest way to think of the n-Lorem foundation is as a bridge that connects the need-the patients with unique mutations, their physician investigators and the institutions caring for the patients-to the ASO discovery and development engine created at Ionis Pharmaceuticals over the

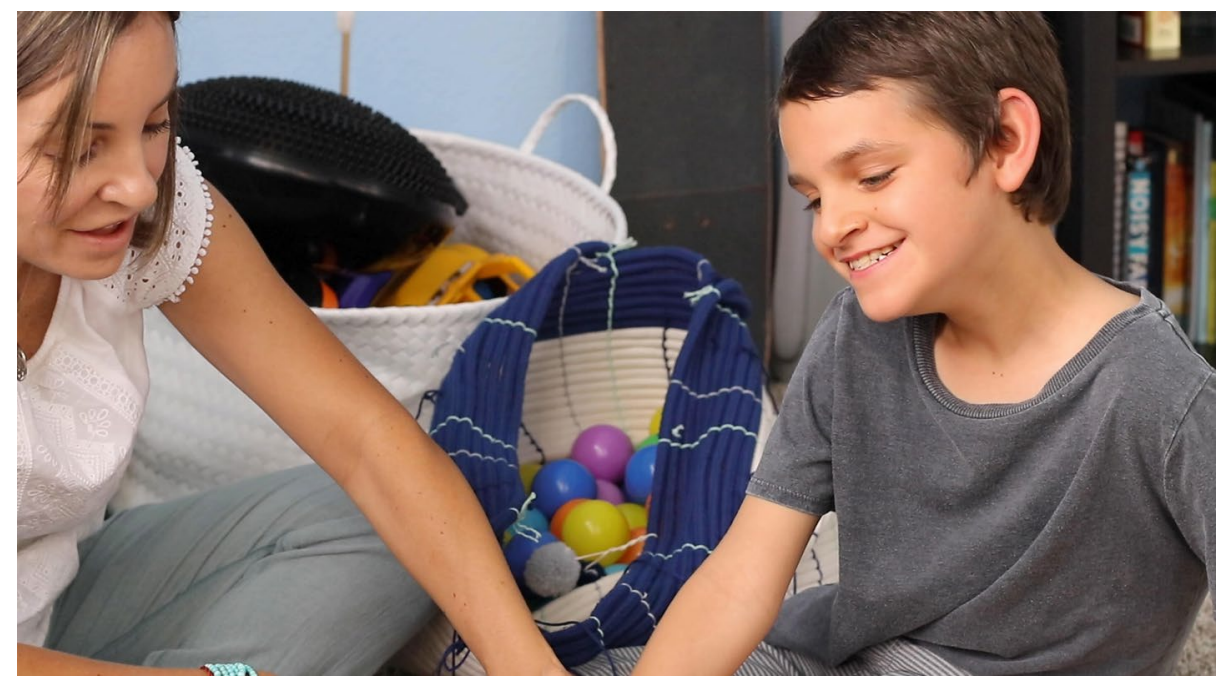

Kelley Dalby with her son Connor, who has been accepted into the n-Lorem program. When he was just 8 months old, Connor was diagnosed with West syndrome, a severe form of epilepsy that rarely responds to medication and is associated with mutations in X-linked CDKL5 or ARX. Source: $\mathrm{n}$-Lorem.

Table 1 | Post-RNA-binding mechanisms of action of phosphorothioate ASOs

\begin{tabular}{|c|c|c|}
\hline \multicolumn{2}{|l|}{ Occupancy only } & \multirow[t]{2}{*}{ Occupancy-induced degradation } \\
\hline Loss of function & Gain of function & \\
\hline 5' cap inhibition & Altered processing & RNase H1 \\
\hline Translational arrest & Upstream open reading frame utilization & $\mathrm{AGO} 2$ \\
\hline \multirow{3}{*}{$\begin{array}{l}\text { Alternate } \\
\text { polyadenylation } \\
\text { signal utilization }\end{array}$} & Translation inhibitory element utilization & Nonsense-mediated decay \\
\hline & Nonsense-mediated decay inhibition & No-go decay \\
\hline & Premature termination codon readthrough & \\
\hline
\end{tabular}

past four decades. One of the first challenges was to create an interface that would open the way to patient referrals from the ultra-rare disease community.

Patients or their parents and research physicians submit substantial patient genetics to qualify for $n$-Lorem experimental ASO treatments through a web interface. The charity has developed processes that assure high-quality risk/ benefit judgements in selecting patients and diseases and making available to patients the highest quality ASOs possible. The first step is to assure that the best advice from all relevant disciplines is considered to determine whether a patient with an ultra-rare mutation is an appropriate one to treat with an ASO. All potential candidates are presented to the Access To Treatment Committee (ATTC), comprising experts in all relevant areas (Table 1). The next step is to develop a specific treatment, natural history and evaluation plan unique to each patient-a process that has never been done before. The $n$-Lorem process assures clarity about what the treatment goals are, how they will be measured; then, during the months required to discover and develop the ASO to treat the patient, the key endpoints are measured so that the incidence and severity 
of these measures during treatment can be compared with pre-treatment values. The last step is, via the collaboration with Ionis, to select the very best possible ASO to treat the patient.

$\mathrm{n}$-Lorem is committed to publishing annually the results of each program. The foundation funds the discovery, development and manufacture of experimental ASOs at Ionis, assuring the highest quality antisense drug is produced under good manufacturing practice (GMP) conditions. It then works with the physician investigator and institution to prepare an investigator-initiated Investigational $\mathrm{New}$ Drug (IND) application to the US Food and Drug Administration (FDA).

\section{Defining ultra-rare disease}

The biopharmaceutical industry's increasing focus on rare diseases has both added to knowledge about these conditions and spurred many new therapies. Today, it is generally accepted that there are over 7,000 rare diseases and it is estimated that about 250 novel diseases are added annually. This number is also likely to increase as more humans are genetically characterized $^{1}$. Although definitions of rare disease differ around the world, the FDA defines it as a malady that affects fewer than 200,000 people in the United States $^{2}$. In contrast, orphan disease is used as an operational term, with the agency defining it as any indication unlikely to be commercially attractive. (In the European Union, orphan diseases are considered to be rare or neglected diseases ${ }^{3}$.) Irrespective of the definition or the regulatory category, in recent decades, the industry has made substantial progress in bringing new therapies to small populations of patients or underserved patient populations. However, even today, it is challenging to define the true incidence and prevalence of rare diseases.

Arguably the most accurate and useful estimates derive from the Orphanet database ${ }^{4}$. This database contains information on over 6,000 unique rare diseases, and the analytical methods employed make the result particularly informative. On the Orphadata platform, $\sim 72 \%$ of rare diseases are genetically caused and almost $70 \%$ manifest as pediatric diseases. Importantly, $84.5 \%$ of the diseases evaluated have point prevalences of less than 1 in $1,000,000$, but nearly $80 \%$ of the population burden is contributed by the $4.2 \%$ of diseases that are the most common. None of these numbers is particularly surprising, as one would expect that the bulk of patients would be contributed by the most common rare diseases. However, the number of diseases that have a point prevalence of less than 1 in 1,000,000 provides a sobering view of how many $N$-of-1 patients there may be, and the total number of rare disease patients is surprisingly high, with estimates of 263-466 million patients worldwide. These numbers provide a glimpse of the scale of the problem presented by ultra-rare diseases.

Ultra-rare diseases are even more difficult to define than rare diseases, either by incidence or operationally. Nor is it possible to clearly define the overall prevalence of ultra-rare diseases or prevalence of specific ultra-rare diseases. However, as medical research institutions and consortia, such as the Undiagnosed Diseases Network (UDN), focus on this area, it is increasingly apparent that many hundreds of thousands to many millions of patients experience severe, often rapidly fatal genetic diseases that frequently result in substantial developmental delays as well. As the number of humans who have undergone extensive genomic characterization increases, the incidence of true ultra-rare diseases identified is increasing exponentially.

\section{The unique challenges of $\mathrm{N}$-of- 1 patients}

Patients with unique or extraordinarily rare disease-causing mutations present a host of extremely challenging issues. Most still simply progress and succumb to these diseases because they are either misdiagnosed or undiagnosed. Those fortunate enough to be referred to a tertiary care center focused on caring for ultra-rare patients typically spend years seeking a diagnosis only to be given a name for their afflictions and genetic cause for their disease but told that there is no treatment and that it is highly unlikely that there will ever be an effective therapy available (for a patient perspective, see Box 1).

This tragic situation is quite simply a product of small numbers. For illnesses that affect 1-30 patients worldwide, it is difficult even to conceive a commercially viable path to prove that an agent is safe and effective in the clinic. Even if a clinical trial could be designed to define the benefit/ risk profile of the new agent sufficiently to achieve commercial approval, the costs of commercial development would mean that the price of therapy would likely be far too great to be afforded.

Moreover, $N$-of- 1 patients typically suffer severe symptoms and often rapidly progress to extreme debilitation and death. As mentioned above, those fortunate enough to be diagnosed typically spend years before a cause of the disease is identified, and during that time their disease worsens. Particularly tragic is the fact that symptoms of many of these mutations manifest during infancy and are associated with severe developmental delays, arrest of development or death. Thus, almost all of these patients demand treatment urgently. Even for patients with inherited diseases, rather than those with sporadic mutations, the patients are typically dispersed across the world's population. This means that diagnosis is extremely difficult and even if a clinical trial could be devised for, say 20-30 patients, recruitment of patients and qualified investigators to conduct such a trial would be extremely difficult, time consuming and expensive. Obviously, for patients who have mutations unique to them (and there are many of these patients), there is no other patient with precisely the same disease. Finally, current regulatory processes are designed to evaluate the effects of new agents on large populations of patients, and even regulations applied to rare populations of patients do not address the issues faced by $N$-of-1 patients.

To meet the needs of patients with unique mutations, at least four elements must be in place. First and foremost, a technology sufficiently efficient and versatile to rapidly and cost-effectively create new personalized treatments for patients who have diverse types of mutations that may affect a wide range of organs is necessary. Second, as discussed above, we need a model that departs from the typical commercial model. In fact, even models successfully applied to bringing commercial treatments to patients with rare diseases are simply not feasible to address the needs of these patients. Third, it is essential that the patient's mutation, the function of the mutated gene and the clinical situation be understood in depth by a clinical investigator who is deeply committed to the patient at an institution capable of managing the patient under an investigator-initiated IND application. Finally, because any process that could lead a therapeutic intervention that may bring benefit to a single patient imposes unique constraints on development, a regulatory environment that is supportive of finding solutions for these patients is a must.

\section{Antisense as a solution}

The intrinsic productivity of ASO technology is now broadly accepted, and the factors resulting in the efficiency of the technology are well understood ${ }^{5,6}$. The efficiency of the technology is further enhanced by the automation and design algorithms available at Ionis, which means that the discovery of the optimal ASO is rapid and inexpensive. Because $N$-of- 1 patients express a wide range of mutations, the need for multiple 


\section{Box 1 | A desperate journey}

Tragically, even when a syndrome is well known and fully understood, typically the journey to a diagnosis is lengthy, arduous and dangerous. A good example of how perilous the journey to a diagnosis can be is represented by a case that has been submitted to n-Lorem.

Almost four years ago, a young couple joyously welcomed a healthy baby girl into their family after an uneventful pregnancy and delivery. Despite the joy and apparent health of their baby, over a very few months the parents became concerned that their child seemed to not be meeting her expected growth and other milestones. Their pediatrician explained the wide variations in when children reach developmental milestones. Nevertheless, as the months passed, it became obvious that something was wrong. They met with numerous specialists as their daughter worsened and required hospitalizations. Sadly, while the child was in a hospital, she had two cardiac arrests and was resuscitated. As the hospital was not equipped to care for such a sick child, she was transferred to a tertiary care facility. She was 3 years old when a diagnosis was finally made.

Cockayne syndrome was first described in 1936 and has since been 'rediscovered', so it has other names as well. There are four clinical subtypes of this disease. The genes responsible are well known and are involved in transcription-initiated excision DNA repair. Thus, patients with these mutations are deficient in clearing single and double strand crosslinks caused by reactive oxygen species ${ }^{18}$. The two genes responsible for the syndrome are Excision Repair Cross Complex 6 (ECCR6) and ECCR8, with mutations in ECCR6 accounting for about $70 \%$ of cases. The overall incidence is 2.5 in 1,000,000 live births, but the prevalence is unknown. Multiple types of mutations, including splice variants, frameshift and nonsense and missense mutations, can contribute. More than 70 different mutations have been identified ${ }^{19}$. There are no known treatments, and most children pass away in the first decade of life.

Sadly, this child has type 2 CS, one of the most severe forms. Symptoms begin in infancy and progress rapidly. Children display progressive microcephaly, fail to thrive, meet very few developmental milestones, and show progeroid features of rapid aging. This child's mutations appear to be unique to her. Whole-genome sequencing revealed a compound heterozygous mutation, one allele of which creates an upstream cryptic splice acceptor leading to a mis-splicing event that produces a truncated, ineffective protein. n-Lorem is identifying an allele-selective ASO that should cause reversion to the correctly spliced mRNA.

When asked what was important to her, the mother replied, "Time, I just want a little more time with her." The father replied, "Hope." The obvious questions are how much of the developmental damage done can be repaired given how far the disease has progressed. Only in the future, as babies are diagnosed promptly and, hopefully, treated equally promptly, will the answer to that question be available.

Table 2 | Current organs amenable to, and routes of delivery for, ASO treatments

\begin{tabular}{lll} 
Organs & Routes & Total annual dose \\
\hline Central nervous system & Intrathecal & $500 \mathrm{mg}$ \\
Liver & Subcutaneous & $200 \mathrm{mg}$ \\
Lung & Aerosol & $3 \mathrm{~g}$ \\
Kidney & Subcutaneous & $5-10 \mathrm{~g}$ \\
Eye & Intravitreal & $<50 \mathrm{mg}$
\end{tabular}

post-RNA-binding mechanisms and the ability to be allele-selective are critically important. Thanks to continuing advances in the ASO platform, the needs of many of these patients can be met as it is now possible to design ASOs to take advantage of multiple post-RNA-binding mechanisms, including those that can selectively increase translation of many proteins (Table 2). Because ultra-rare mutations can occur in any cell and affect the performance of any organ, the capacity to administer phosphorothioate ASOs by multiple routes of delivery ${ }^{5,7-9}-$ either systemic (intravenous, intramuscular, subcutaneous and oral) or local (intravitreal, intrathecal, aerosol and rectal) _ and distribute them broadly enhances the number and types of patients who qualify for treatment.

To optimize benefit versus risk, today, $\mathrm{n}$-Lorem is focusing on the routes of administration and organs shown in Table 3. Given that phosphorothioate ASOs show high potency and duration of action in these organs by these routes of administration, together with proven stability, the cost of manufacturing sufficient ASOs to treat a single patient for many years is modest.

Two more factors are critical to facilitating development while minimizing risks to a patient being treated with an entirely new experimental agent. The consistent behaviors of each individual ASO within a given chemical class, coupled to the detailed understanding of the routes of delivery, distribution and potency in cells in the organs on which n-Lorem is focusing, facilitate the selection of the route of administration, the dose and the dose frequency.

Finally, Ionis has created databases and published findings that integrate all safety observations from non-human primates through all controlled clinical trials for all the chemical classes in use at the company. These databases greatly facilitate the assessment of potentially drug-related safety events $^{5,7-9}$. Table 3 presents the attributes of ASO technology that are critical to meeting the needs of patients with ultra-rare diseases. The first and by far the most important collaboration is the alliance with Ionis.

\section{Why a charitable model?}

In the United States, the development and commercialization of ASOs are regulated by the FDA's Center for Drug Evaluation and Research. Although the FDA is developing detailed guidance for the use of ASOs in patients with ultra-rare mutations and has published initial administrative guidance, there is no path to approval for an agent that is effective in a single patient.

If the FDA develops approaches that might result in limited approval or an approach that would provide a new category different from an IND under which long-term treatment might be regulated, n-Lorem will respond appropriately. Furthermore, even if a path to commercial approval were ultimately developed, the most modest development process in a commercial context would likely result in extremely high annual costs to treat individual patients. For these reasons, we developed $n$-Lorem using a charitable model.

\section{Access to genomic information and clinical expertise}

The UDN is a US National Institutes of Health consortium of tertiary care centers 
Table 3 | Attributes of ASO technology that facilitate $\mathrm{N}$-of-1 treatments

\begin{tabular}{ll}
\hline Attribute & Further details \\
\hline Efficiency of drug discovery and development & See main text "Antisense as a solution" and refs. ${ }^{5,7-9}$ \\
\hline Versatility & Multiple post-binding mechanisms (see Table 1) \\
& Multiple routes of administration (see Table 2) \\
& Multiple organs (see Table 3) \\
\hline lonis automation & See main text "Antisense as a solution" \\
\hline Performance consistency in chemical classes & Potency (see Table 3) \\
& Dose frequency (weekly to semi-annually, depending \\
& on route of administration) \\
\hline Integrated safety databases & Adverse events ${ }^{14-17}$ \\
\hline $\begin{array}{l}\text { Potency of modern chemistries in selected } \\
\text { organs }\end{array}$ & See refs. ${ }^{14-17}$ \\
\hline Low cost of goods & See Table 2 \\
\hline
\end{tabular}

that has for several years focused on achieving diagnoses and characterizing the genetic causes of disease in patients who display rare clinical syndromes that have been difficult to assign to a particular disease. These institutions perform further research to characterize the function of the gene that is mutated. The UDN endeavor provides clear evidence that many previously unidentified diseases can be diagnosed and the genetic causes of such diseases determined with a focused effort. The UDN experience also demonstrates (i) that novel mutations that differ from the main genetic cause of a rare disease are frequent, (ii) that many different clinical phenotypes are not yet included in known diseases, and (iii) that de novo unique mutations are more common than previously appreciated. Moreover, because UDN institutions are centers of research, they also provide potential clinical investigators who are capable of treating a patient with an experimental ASO. In essence, the UDN institutions provide all the elements necessary for n-Lorem to provide experimental ASOs. Thus, the UDN is an important collaboration and has been a model for relationships with other tertiary care centers.

As important as the UDN institutions are in providing the up-front components to the ultimate treatment of an $N$-of- 1 patient, our first year experience has shown that many institutions that are not formally a part of the UDN are also focused on addressing the needs of ultra-rare patients and are taking approaches similar to those used by the UDN. In fact, less than half of the applications for treatment have come from UDN institutions. This constitutes important news for patients with ultra-rare diseases as n-Lorem considers all the tertiary-care institutions with which we work as partners working together to bring treatment to these patients.

\section{A supportive regulatory environment} In early 2019, n-Lorem initiated discussions with the senior leaders of the FDA Center for Drug Evaluation and Research and received a rapid and supportive response. In subsequent meetings, foundation leadership introduced the n-Lorem concept more broadly within the FDA and learned that the agency was developing guidance for the treatment of patients with ultra-rare diseases. During the initial opportunity for public comment, n-Lorem submitted a document that suggested several potential steps that the FDA might take to facilitate meeting the therapeutic needs of such patients. One recommendation was that the agency establish a process that supports informal interactions when the decision to treat a patient is made to assure that the preclinical development plan is agreed upon. It was also recommended that the INDs for patients to receive experimental ASOs be managed by a single division of the FDA, thus enhancing the consistency of regulatory processes. n-Lorem also proposed rapid and limited preclinical development that certainly would not be adequate for formal approval of any agent, but sufficient, given the knowledge about ASOs, to assure that patients are exposed to only prudent risks. Several other proposals pertained to manufacturing and stability testing.

Recently, the FDA has issued initial administrative guidance for the development of ASOs for patients with ultra-rare diseases and asked for comments. As n-Lorem awaits more detailed guidance, it will continue to keep the agency aware of progress. The charity has already had the opportunity to work with two investigators to provide experimental ASOs to several patients; that experience gives optimism that FDA's definitive guidance will be supportive of n-Lorem's approach.

With all the above aspects now in hand-a philanthropic model, ASO drug progress, human genomic information, clinical expertise and regulatory engagement $-\mathrm{n}$-Lorem has initiated a concerted drug development effort to address ultra-rare diseases. Here I detail progress made in putting into practice a model to realize the goal of $N$-of- 1 treatments at the foundation.

\section{Founding and launching $\mathbf{n}$-Lorem}

The founding donors of $n$-Lorem were Ionis Pharmaceuticals and Biogen, together with Rosanne Crooke and I. To complement the donor funding, Ionis provides limited contributions in kind and services to $\mathrm{n}$-Lorem via a fee-for-service contract. Many volunteers at Ionis provide services gratis as well.

Given the novelty of the foundation, the plan was to seek more donations only after the concept was shown to be workable, perhaps in late 2021. In fact, given the success that $\mathrm{n}$-Lorem experienced collaborating as we were forming $\mathrm{n}$-Lorem with two investigators, Tim Yu of Harvard Medical School and Neil Shneider of the Columbia School of Medicine; the substantial number of applications for treatment; other progress; and the enthusiastic responses from many, a large number of donors were added in 2020 (Table 4). The charity is now preparing to engage in formal fundraising that will be necessary to meet the substantial demand for experimental ASO treatment.

Despite 2020 being a challenging year for many reasons and n-Lorem being a nascent effort attempting to bring experimental ASO treatments to patients who, to a tragically large extent, have never been treated effectively, excellent progress was made in seven main areas summarized below.

Supporting clinical investigators by
providing experimental ASOs to patients
with ultra-rare mutations. The experience
gained from helping two investigators obtain
experimental ASOs for several patients has
been invaluable. Based on these collaborative
efforts, we can conclude that there is a rapid
and cost-effective path toward creating and
providing experimental ASOs charitably and
that, even in patients suffering from diseases
that rapidly progress to death, it is possible
to identify and provide an experimental
ASO. Conversations with the FDA also
provide n-Lorem leadership with optimism
it will be effective when detailed guidance
for experimental ASO treatment of patients


Table 4 | ATTC expertise for evaluating whether a patient should be treated with an experimental ASO

\begin{tabular}{|c|c|}
\hline Expertise & ATTC members \\
\hline $\begin{array}{l}\text { Antisense } \\
\text { technology }\end{array}$ & $\begin{array}{l}\text { Frank Bennett, lonis Pharmaceuticals, Carlsbad, CA, USA. } \\
\text { Kristina Bowyer, lonis Pharmaceuticals, Carlsbad, CA, USA. } \\
\text { Matt Buck, lonis Pharmaceuticals, Carlsbad, CA, USA. } \\
\text { Eugene Schneider, lonis Pharmaceuticals, Carlsbad, CA, USA. } \\
\text { Walter Singleton, lonis Pharmaceuticals, Carlsbad, CA, USA. } \\
\text { Eric Swayze, lonis Pharmaceuticals, Carlsbad, CA, USA. }\end{array}$ \\
\hline Genetics & $\begin{array}{l}\text { Manisha Balwani, Icahn School of Medicine at Mount Sinai, New York, NY, USA. } \\
\text { Alan Beggs, Harvard Medical School, Cambridge, MA, USA. } \\
\text { F. Sessions Cole, Washington University School of Medicine, St. Louis, MO, USA. } \\
\text { Darryl De Vivo, Columbia University, New York, NY, USA. } \\
\text { Joseph Gleeson, Rady Children's Institute for Genomic Medicine, San Diego, CA, USA. } \\
\text { Matthias Kretzler, University of Michigan, Ann Arbor, MI, USA. } \\
\text { Jeffrey L. Noebels, Baylor College of Medicine, Houston, TX, USA. } \\
\text { Tim Yu, Boston Children's Hospital, Harvard Medical School, Boston, MA, USA. } \\
\text { Jeff Carroll, Western Washington University, WA, USA; independent consultant to } \\
\text { n-Lorem. } \\
\text { Frank Bennett }\end{array}$ \\
\hline $\begin{array}{l}\text { Relevant } \\
\text { disease } \\
\text { expertise }\end{array}$ & $\begin{array}{l}\text { Richard Finkel, St. Jude Children's Research Hospital Memphis, TN, USA. } \\
\text { Willis Maddrey, Willis C Maddrey Consulting LLC, Plano, TX, USA. } \\
\text { Alfred Sandrock, Biogen, Cambridge, MA, USA. } \\
\text { Eugene Schneider } \\
\text { Manisha Balwani } \\
\text { Alan Beggs } \\
\text { F. Sessions Cole } \\
\text { Darryl De Vivo } \\
\text { Joseph Gleeson } \\
\text { Matthias Kretzler } \\
\text { Jeffrey L. Noebels } \\
\text { Tim Yu }\end{array}$ \\
\hline $\begin{array}{l}\text { Clinical care } \\
\text { expertise }\end{array}$ & $\begin{array}{l}\text { Manisha Balwani } \\
\text { Alan Beggs } \\
\text { F. Sessions Cole } \\
\text { Darryl De Vivo } \\
\text { Joseph Gleeson } \\
\text { Willis Maddrey } \\
\text { Matthew Might } \\
\text { Jeffrey L. Noebels } \\
\text { Tim Yu } \\
\text { Richard Finkel }\end{array}$ \\
\hline $\begin{array}{l}\text { Clinical trial } \\
\text { management }\end{array}$ & $\begin{array}{l}\text { Tiffany Sepp, Vanguard Clinical, Solana Beach, CA, USA; consultant to n-Lorem } \\
\text { Walter Singleton } \\
\text { Eugene Schneider } \\
\text { Willis Maddrey } \\
\text { Tim Yu } \\
\text { Matthias Kretzler } \\
\text { Alfred Sandrock }\end{array}$ \\
\hline Regulatory & $\begin{array}{l}\text { Lauren Black, Charles River Laboratories, Washington, DC, USA. } \\
\text { Matt Buck } \\
\text { Willis Maddrey }\end{array}$ \\
\hline Bioethics & $\begin{array}{l}\text { Matthew Might, Precision Medicine Institute at the University of Alabama, } \\
\text { Birmingham, AL, USA.. } \\
\text { Hugh Kaul, Precision Medicine Institute at the University of Alabama, Birmingham, } \\
\text { AL, USA. }\end{array}$ \\
\hline $\begin{array}{l}\text { Charitable } \\
\text { foundation }\end{array}$ & Tracy Johnson, n-Lorem, Carlsbad, CA, USA. \\
\hline
\end{tabular}

with ultra-rare mutations is published by the agency. In addition, our experience will be of interest to the FDA as guidance is developed.
Establishing quality systems. n-Lorem had to establish systems to assure that complex risk/benefit decisions are made thoughtfully in a manner that optimizes the opportunity for benefit while exposing patients only to prudent risks. The charity's website describes its mission and provides a site to which research physicians and patients or parents can apply for treatment, with clear instructions as to the information required. It also provides investigators with guidance about what is required from them and their institutions. Applications are blinded with regard to patient identity, then subjected to a rigorous evaluation process that include presentation of the case to a committee (the ATTC) comprising all the expertise needed to assess whether an experimental ASO is appropriate for the patient (Table 1).

For the discovery and development of experimental ASOs, $\mathrm{n}$-Lorem depends on the quality systems developed over the past three decades at Ionis, including proven high-quality ASO discovery and design, toxicological studies, and manufacturing and analytical processes. We are standardizing to a large extent the information included in investigator-initiated INDs as well and have defined metrics to measure the overall performance of n-Lorem.

Evaluating performance. $n$-Lorem is committed to evaluating overall performance of the charity's effort, evaluating the performance of individual ASO programs in the patient, and publishing our results annually in the biomedical literature (Table 5). Key parameters of success for $n$-Lorem are the number of applications received, the fraction of applications amenable to experimental ASO treatment, the time to decision on a particular program, and the time to treatment of a patient.

In contrast to metrics to measure the overall performance of $n$-Lorem, the evaluation of the performance of individual ASOs creates novel challenges. Several efforts to design a clinical study for single patients have been reported in the literature. All of these approaches use a crossover design that involves treating the patient for a period of time with the experimental agent, then crossing over to placebo or established agent $^{10-13}$. At n-Lorem, we have chosen a different approach for several reasons.

First, ASOs have a long duration of action, making a crossover design impractical. Second, n-Lorem patients are typically quite ill and usually have no other agents available for comparison, resulting in the need to compare with placebo, which the n-Lorem team believes is generally untenable. Finally, the programs do not seek approval as the plan is to provide experimental ASOs to a patient for free for life under investigator-initiated INDs. 
Obviously, should the FDA guidance include a path to approval, this position will be reevaluated.

The strategy to evaluate the performance of each ASO in each patient is patient centered. Before treatment, the n-Lorem chief medical officer works with the investigator to define a treatment plan that identifies primary, secondary and exploratory treatment goals and methods of measurement. Then, during the period of time required to discover and develop the ASO, the investigator and patient or parent execute the agreed natural history plan using the measures that will be assessed during treatment. This assures that there are recent detailed data on the patient's condition to which to compare to the patient's condition during treatment.

\section{Establishing collaborations to enhance} patient access and reduce treatment cost per patient. In n-Lorem's first year of operation, a key focus was to establish collaborations that assure as many patients as possible have access to treatment and to reduce costs. Given the importance of the UDN to the diagnosis and genetic characterization of disease in patients with ultra-rare mutations, a formal collaboration agreement has been established with the UDN. However, n-Lorem considers all institutions that are focused on these unique patients and that submit applications for treatment to be important informal collaborators and may establish additional formal collaborations with non-UDN tertiary care institutions. Given that the major monetary expenditure for programs is the cost of toxicological studies, n-Lorem is grateful to all of the contract research organizations that have agreed to perform such studies at substantially reduced costs. The Korea Institute of Toxicology, Covance and Charles River Laboratories are contributing additional services, and Charles River Laboratories has also donated money to the charity. As many applications include inadequate information about the mutation to support a decision to treat, Illumina has contributed genomic sequencing services as well.

\section{Broadening awareness of $\mathbf{n}$-Lorem.}

Because n-Lorem is a novel concept and was unknown, a key task for 2020 was to broaden awareness across several audiences. It was essential that physicians and patients who have ultra-rare mutations that affect the performance of any of the five organs (Table 2) on which n-Lorem is focusing be aware that they now have an opportunity for treatment. To that end, $\mathrm{n}$-Lorem has made presentations to many tertiary care centers and appropriate subspecialty societies, with more scheduled. We have also made presentations to several disease-focused patient advocacy groups, but given the number of these groups, much remains to be accomplished. Furthermore, presentations to professional organizations focused on genetic diseases and industry organizations have been made, with still others scheduled. In short, solid progress in assuring awareness of n-Lorem has been achieved, but vastly more remains to be accomplished.

Applications to treat. Given the novelty of $n$-Lorem and the impact of COVID-19 and lockdowns, expectations concerning the number of applications were low, with an anticipation of perhaps 5 applications in 2020. In fact, a total of 50 applications were submitted to the charity website (Table 5). On the basis of the trajectory of applications, we expect to receive more than 75 new applications in 2021. Fortunately, ASO technology is expandable to meet the demand if sufficient funds are made available. Nearly all the applications to treat have been from neurologists and involve diseases primarily manifesting in the central nervous system; however, the charity has received more recent patient applications to treat diseases that manifest in the kidney, so we expect that both the number and diversity of applications will increase substantially in 2021.

Although ASO antisense technology is versatile, it is not a panacea. In particular, null mutations are not typically amenable to treatment with ASOs, and if the specific genetic cause is unclear, it is unwise to expose a patient to intervention. Thus, the expectations at n-Lorem are that at steady state, perhaps $10-15 \%$ of applicants will be amenable to experimental ASO treatment. To date, however, $\sim 35-40 \%$ of applicants have been accepted for treatment.

Working toward well-defined regulatory guidance. Charitably providing experimental ASO treatment for patients with ultra-rare diseases is a concept not contemplated by existing regulatory processes or guidance. Although it has been possible to work within the existing regulatory structure to provide ASOs to a few patients, it is very important that more definitive and specific regulatory process and guidance be developed, including guidelines about the minimum preclinical data required and the overall means by which this effort is regulated. The n-Lorem team recognizes that any approach to the treatment of these patients must be done professionally and in a manner that assures that patients are exposed to only prudent

\section{Table 5 | n-Lorem overall performance metrics in $\mathbf{2 0 2 0}$}

Performance metric

2020 data

$\begin{array}{ll}\text { Accepted case submissions } & 16 \\ \text { Pending case submissions } & 12 \\ \text { Denied case submissions }^{\mathrm{a}} & 22\end{array}$

Average time from submission 50 days to decision by ATTC

Average time from submission to treatment

To be determined

${ }^{\circ}$ Cases are denied if the mutation is unapproachable with ASO technology or expressed in an organ beyond n-Lorem's current focus, or if an investigator or institution is unable to file an IND.

risks and that risk/benefit decisions are made appropriately. In this respect, it is encouraging that initial guidance has already been issued by the FDA, and the charity plans to provide an additional response to the recommendation it submitted to the agency in 2020. Overall, n-Lorem plans to work closely with the FDA to create definitive regulatory guidelines that will facilitate the provision of experimental ASO treatments to the appropriate patients.

\section{Current focus and future perspective}

Ultra-rare disease affects millions of patients worldwide, and yet it has been largely neglected by traditional drug discovery and development programs until now as a result of a lack of commercial feasibility. In the first year since its founding, $\mathrm{n}$-Lorem has made substantial progress in providing a new treatment paradigm for a set of patients affected with these conditions. To provide a sense of the complexity of patients with ultra-rare mutations, in Table 6, I provide some examples of patients with mutations that cause neurological disease. The number of applications received by $n$-Lorem (Table 5) demonstrates the pent-up need for solutions that can benefit some of the patients suffering from ultra-rare diseases and shows that patient recruitment via the charity's approach can work. In 2021, n-Lorem intends to build on its success with an exciting and challenging agenda.
Advancing ASO treatments for n-Lorem patients. The single most important objective is to advance ASOs for the $\sim 20$ patients approved for treatment. The volunteers who have supported n-Lorem have met those needs so far. However, given the demand, paid employees must be recruited to perform the necessary work and expand the reach of $\mathrm{n}$-Lorem. 
Table 6 | Details of selected $\mathbf{n}$-Lorem programs under development

\begin{tabular}{lllll} 
Gene & Treating physician & Patient age, sex & Chief symptoms & ASO strategy \\
\hline SCN2A & Joe Gleeson & 10, male & Seizures, developmental delay & Allele-selective RNase H \\
GNAO1 & Brenda Porter & 2.5 , male & Seizures, neurodegeneration & Allele-selective RNase H \\
GNAO1 & William Gallentine & 2, female & Seizures, neurodegeneration & Allele-selective RNase H1 \\
KCNQ2 & Deborah Holder & 5, female & Seizures & Allele-selective RNase H \\
SPAST & Steven Wolf & 16, male & Spasticity, paraplegia & Allele-selective RNase H \\
ERCC6 & Joe Gleeson & 3, female & Progeria, failure to thrive, developmental delay & Splicing ASO \\
SLC6A7 & Scott Demarest & 3, male & Developmental delays, seizures, behavioral issues & $\begin{array}{l}\text { Generating data demonstrating functional } \\
\text { via upstream open reading frame ASO }\end{array}$ \\
\hline
\end{tabular}

additional genomics characterization in process.

In response, we are preparing to engage in formal fund-raising efforts. As these patients are treated, the performance of the systems developed to evaluate the benefits of ASOs in each of the patients will be assessed, approaches refined on the basis of our experience, and the results published.

Expanding awareness of $\mathbf{n}$-Lorem and managing increased demand. A substantial increase in the number and diversity of applications received in 2021 is expected, and funding has been obtained so that we may expand to meet the needs of those patients. Further work must be carried out to assure that all relevant medical subspecialties are aware of $\mathrm{n}$-Lorem and to develop relationships with more patient advocacy groups to again assure that as many patients with various types of ultra-rare diseases are aware of the opportunity presented by our charitable foundation, including cautious expansion of staff at $\mathrm{n}$-Lorem to meet this demand.

Broaden the network of collaborations. To be fully successful, meeting the needs of patients with ultra-rare diseases must be a collaborative effort. n-Lorem will continue to work toward adding as collaborators chief medical officers who can supply experimental ASOs under good manufacturing conditions to lower that cost even further. We hope to add other biotech and pharmaceutical companies as donors and collaborators. We continue to encourage industry associations to partner with $\mathrm{n}$-Lorem as we believe that it is in the interest of the industry to bring the benefits of new technology and medicines to patients with ultra-rare diseases. As other technologies mature to the point that they can be used charitably to approach the needs of patients not amenable to ASO treatment, we will seek to collaborate with providers of them as well.
Initiating formal fundraising activities. The single limit to meeting the needs of patients with ultra-rare diseases that are amenable to experimental ASO treatment is financial. The foundation is buoyed by the community's enthusiastic response and the number of new donors added in 2020, but in 2021 fundraising efforts must be formalized and redoubled.

\section{Working with FDA to establish definitive} guidance. Definitive supportive regulatory guidance is urgently needed. However, until formal full guidance is published, n-Lorem and these patients will be in an undefined regulatory environment. In 2021, the charity looks forward to continued productive engagement with the FDA in the hope that definitive guidance can be released.

\section{Conclusions}

The $n$-Lorem charity is a novel experiment in meeting the needs of patients with ultra-rare diseases. Progress recorded during the first year of operation has been rapid, but $n$-Lorem remains a nascent effort; even if it succeeds in meeting initial expectations, the effort to harness the power of modern technologies to serve the needs of these desperate patients is just beginning. I believe that the needs of ultra-rare disease sufferers can be best met only if all stakeholders collaborate. Once again, on the basis of the progress registered in n-Lorem's first year, I am optimistic that many other organizations will join in this effort. As other technologies mature, I look forward to working with the practitioners of those approaches either as partners in n-Lorem or in parallel efforts with effective integration to assure that as many patients are served as possible. Clearly, all involved are learning as they go, so enhancements to the foundation's systems and approaches based on initial experiences are to be expected. The n-Lorem foundation cannot go it alone and is looking forward to working with all interested parties to bring hope and treatments to these underserved patients. I look forward to reporting regularly on progress.

\section{Stanley T. Crooke ${ }^{凶}$}

$n$-Lorem and Ionis Pharmaceuticals, Carlsbad,

CA, USA.

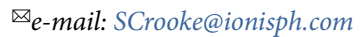

Published online: 4 June 2021

https://doi.org/10.1038/s41587-021-00945-0

References

1. Nguengang Wakap, S. et al. Eur. J. Hum. Genet. 28, 165-173 (2020).

2. Jo, A. et al. BMJ Open $9, \mathrm{e} 027248$ (2019).

3. Copley-Merriman, K. Value Health 21, 491-492 (2018).

4. Richter, T. et al. Value Health 18, 906-914 (2015).

5. Crooke, S. T., Seth, P. P., Vickers, T. A. \& Liang, X. H. J. Am. Chem. Soc. 142, 14754-14771 (2020).

6. Crooke, S. T., Liang, X. H., Crooke, R. M., Baker, B. F. \& Geary, R. S. Biochem. Pharmacol. 2020, 114196 (2020).

7. Crooke, S.T., Baker, B.F., Crooke, R.M. \& Liang, X.H. Nature Rev. Drug Discov. https://doi.org/10.1038/s41573-021-00162-z (2021)

8. Crooke, S.T., Liang, X.H., Baker, B.F. \& Crooke, R.M. J. Biol. Chem. https://doi.org/10.1016/j.jbc.2021.100416 (2021).

9. Crooke, S. T., Vickers, T. A. \& Liang, X. H. Nucleic Acids Res. 48 , 5235-5253 (2020).

10. Margolis, A. \& Giuliano, C. Epilepsy Behav. Rep. 12, 100336 (2019).

11. Kravitz, R. L., Sim, I. \& Duan, N. JAMA Intern. Med. 179, 453-453 (2019).

12. Lillie, E. O. et al. Per. Med. 8, 161-173 (2011).

13. Duan, N., Kravitz, R. L. \& Schmid, C. H. J. Clin. Epidemiol. 66 (Suppl.), S21-S28 (2013).

14. Crooke, S. T. et al. Mol. Ther. 24, 1771-1782 (2016).

15. Crooke, S. T. et al. Nucleic Acid Ther. 27, 121-129 (2017).

16. Crooke, S. T. et al. Nucleic Acid Ther. 28, 10-22 (2018).

17. Crooke, S. T. et al. Nucleic Acid Ther. 29, 16-32 (2019).

18. Wilson, B. T. et al. Genet. Med. 18, 483-493 (2016).

19. Vessoni, A. T., Guerra, C. C. C., Kajitani, G. S.,

Nascimento, L. L. S. \& Garcia, C. C. M. Genet. Mol. Biol. 43

(Suppl. 1), e20190085 (2020)

\section{Acknowledgements}

I thank our donors, collaborators, volunteers, board of directors and Access To Treatment Committee members. Thanks also to Kim Butler for excellent assistance and Rosanne Crooke for editorial comment.

Competing interests

S.T.C. is executive chairman of the Board of Directors at Ionis Pharmacueticals, Inc. 